Internat. J. Math. \& Math. Sci.

Vol. 24, No. 4 (2000) 283-288

S0161171200003136

(C) Hindawi Publishing Corp.

\title{
DYNAMICS OF A CERTAIN SEQUENCE OF POWERS
}

\author{
ROMAN SZNAJDER and KANCHAN BASNYAT
}

(Received 14 September 1998 and in revised form 15 January 1999)

\begin{abstract}
For any nonzero complex number $z$ we define a sequence $a_{1}(z)=z, a_{2}(z)=$ $z^{a_{1}(z)}, \ldots, a_{n+1}(z)=z^{a_{n}(z)}, n \in \mathbb{N}$. We attempt to describe the set of these $z$ for which the sequence $\left\{a_{n}(z)\right\}$ is convergent. While it is almost impossible to characterize this convergence set in the complex plane $\mathscr{b}$, we achieved it for positive reals. We also discussed some connection to the Euler's functional equation.
\end{abstract}

Keywords and phrases. Power sequence, dynamics, convergence.

2000 Mathematics Subject Classification. Primary 26A18, 39B12; Secondary 11B37, 37A45.

1. Introduction. In this paper, for any $z \in \mathscr{C} \backslash\{0\}$, we consider the sequence

$$
a_{1}(z)=z, \quad a_{2}(z)=z^{z}=z^{a_{1}(z)}, \ldots, a_{n+1}(z)=z^{a_{n}}(z), \quad n=1,2, \ldots
$$

We wish to characterize the convergence set $L:=\left\{z \in \mathscr{C} \backslash\{0\}:\left\{a_{n}(z)\right\}\right.$ is convergent $\}$. Since $1 \in L$, this set is nonempty. For a complex value of $z$, the mapping $z \rightarrow a_{n}(z)$ is a multivalued operation and $a_{n}(z)$ usually represents an infinite set. If we agree to use the main branch of the complex logarithm (as it is done, e.g., in MATLAB), the above exponentiation becomes a single-valued operation. For arbitrary $z$, it is very easy to run out of accuracy of any available computational software in a few iterations. Thus, it is practically impossible to describe the convergence set $L$. Also, one can show that the sequence $\left\{a_{n}(z)\right\}$ cannot be obtained as a functional iteration, i.e., there exists no function $f: \mathscr{b} \rightarrow \mathscr{b}$ such that $a_{n}(z)=f^{[n]}(z)=f \circ f \circ \cdots \circ f(z)$ (n-times). This sequence has nasty numerical features. Nevertheless, the situation is not hopeless when it comes to considering real values of $a_{n}(z)$.

2. Main result. Our main goal is to demonstrate the following.

THEOREM 2.1. We have $L \cap \mathbb{R}_{+}=\left[e^{-e}, e^{1 / e}\right]$.

Before we proceed with the proof of Theorem 2.1, the following lemma is in order.

LEMmA 2.2. For the function $f(t)=\ln (t) / t$ defined on $\mathbb{R}_{+}=(0,+\infty)$, the following hold true:

(a) range $(f)=(-\infty, 1 / e]$

(b) for any $0<c<1 / e$, the equation $f(t)=c$ has exactly two solutions, while for $c=1 /$ e exactly one, namely $t^{*}=e$.

Proof. The first derivative of the function $f(t)$ is $f^{\prime}(t)=(1-\ln (t)) / t^{2}$, so that $f^{\prime}(t)=0$ if and only if $t=e, f$ is increasing on $(0, e]$ and decreasing on $[e,+\infty)$. Since 
$\lim _{t \rightarrow 0^{+}} f(t)=-\infty$ and $\lim _{t \rightarrow+\infty} f(t)=0$, we obtain statement (a). Since $t^{*}=e$ is the only maximizer of $f(t)$ in $(0,+\infty)$, (b) follows from the intermediate value theorem and the fact that $\lim _{t \rightarrow+\infty} f(t)=0$. The proof is complete.

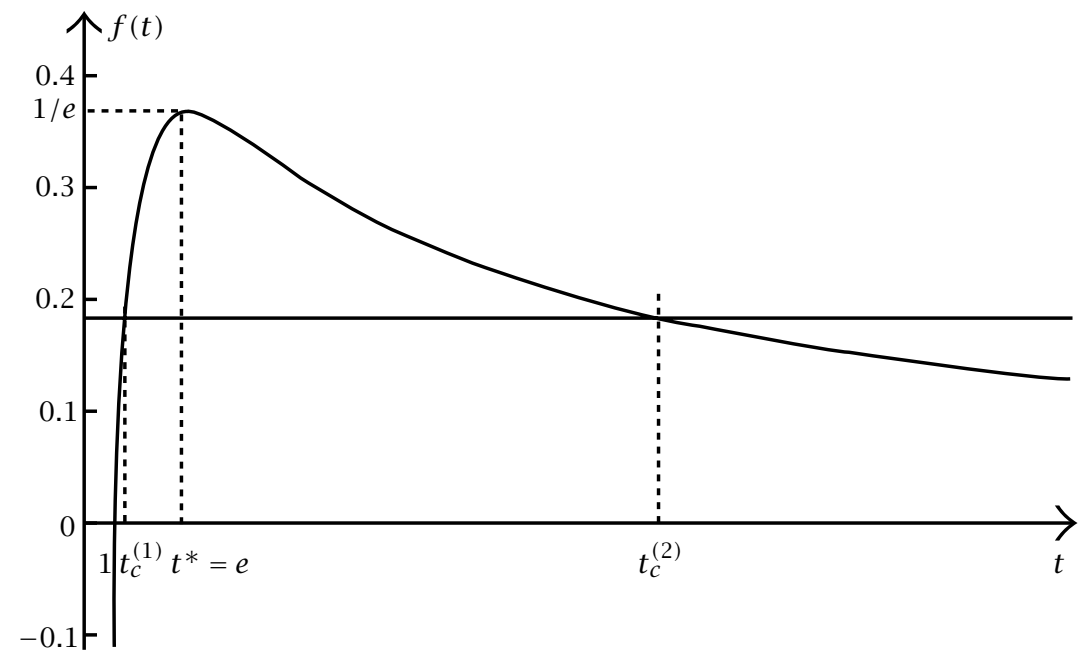

FIGURE 2.1.

Note that if $t_{c}^{(1)}$ and $t_{c}^{(2)}$ denote two distinct solutions of the equation $f(t)=c$, then $t_{c}^{(1)} \rightarrow 1^{+}$and $t_{c}^{(2)} \rightarrow+\infty$ as $c \rightarrow 0^{+}$. This remark follows from the piecewise monotonicity of the function $f(t)$ and the fact that $\lim _{t \rightarrow 1} f(t)=0$ and $\lim _{t \rightarrow+\infty} f(t)=$ 0 . By putting $c=\ln (x)$ in Lemma 2.2, we get the following.

COROLLARY 2.3. For any given $x \in\left(1, e^{1 / e}\right)$ there exist exactly two solutions of the equation $x^{\alpha}=\alpha$, while for $x=e^{1 / e}$, exactly one.

LEMMA 2.4. Let $K(x):=\left\{0 \leq t \leq 1: h_{x}(t)=0\right\}$, where $h_{x}(t)=x^{x^{t}}-t$ and $x>0$. The following statements hold true:

(a) For $x \in\left(0, e^{-e}\right), K(x)$ consists of three distinct elements.

(b) For $x \in\left[e^{-e}, 1\right), K(x)$ is a singleton.

Proof. Instead of giving a rigorous analytic proof similar to Bomberger [1], we rather illustrate our point with an aid of appropriate graphs.

CASE $1\left(x \in\left(0, e^{-e}\right)\right)$. See Figure 2.2.

CASE $2\left(x=e^{-e}\right)$. See Figure 2.3.

In fact, $h_{x}^{\prime}(t)=x^{t} x^{x^{t}}(\ln (x))^{2}-1$, so $h_{e^{-e}}^{\prime}(1 / e)=0$. Also, $h_{e^{-e}}(1 / e)=0$.

CASE $3\left(x>e^{-e}\right)$. See Figure 2.4.

This completes our proof.

PROOF OF THEOREM 2.1

CASE $1\left(0<x<e^{-e}\right)$. By Lemma 2.4 (see Figure 2.2), there exist numbers $0<\alpha_{1}<$ 
$\alpha_{2}<\alpha_{3}<1$ such that

$$
x^{x^{\alpha_{i}}}=\alpha_{i} \text { for } i=1,2,3
$$

While $\alpha_{2}$ is the fixed point of the function $g_{x}(t)=x^{t}, \alpha_{1}$ and $\alpha_{3}$ are points of period $2\left(x^{\alpha_{1}}=\alpha_{3}\right.$ and $\left.x^{\alpha 3}=\alpha_{1}\right)$.

Generating the sequence $\left\{a_{n}(x)\right\}$ by putting $a_{1}(x):=x=g_{x}(1)$ and $a_{n+1}(x)=$ $g_{x}\left(a_{n}(x)\right)$ for $n \geq 1$, as illustrated by Figure 2.5, we get

$$
a_{1}(x)<a_{3}(x)<\cdots<a_{2 n-1}(x)>\alpha_{1}, \quad a_{2}(x)>a_{4}(x)>\cdots>a_{2 n}(x) \succ \alpha_{3} .
$$

Thus, $\left\{a_{n}(x)\right\}$ diverges but consists of two complementary convergent subsequences.

CASE $2\left(x=e^{-e}\right)$. In this case, $\left\{a_{n}(x)\right\}$ is convergent and the limit is $1 / e$. Indeed, the sequence $\left\{a_{n}(x)\right\}$ consists of two complementary subsequences and each of them is convergent to a point of period 2. But in this case, $\alpha_{1}=\alpha_{2}=\alpha_{3}=1 / e$, and the two points of period 2 collapse to the fixed point of the function $g_{e^{-e}}(t)$. Hence, $\left\{a_{n}(x)\right\}$ converges to $1 / e$. However its convergence is very slow (e.g., $a_{1,000,000}\left(e^{-e}\right) \approx$ 0.36697888108297 and $\left.\left|a_{1,000,000}\left(e^{-e}\right)-1 / e\right| \approx 0.00180\right)$.

CASE $3\left(e^{-e}<x \leq 1\right)$. For $x<1$ we reason the same way as in Case 2, so the sequence $\left\{a_{n}(x)\right\}$ converges to a unique fixed point of the function $g_{x}(t)$. For $x=1$, the statement is trivial.

CASE $4\left(1<x<e^{1 / e}\right)$. If $\left\{a_{n}(x)\right\}$ is convergent to a limit $\alpha,\left\{a_{n+1}(x)\right\}$ also converges to $\alpha$, moreover, $a_{n+1}(x)=x^{a_{n}(x)}$ converges to $x^{\alpha}$. Consequently, $x^{\alpha}=\alpha$. By Corollary 2.3, there are exactly two fixed points $\alpha_{1}$ and $\alpha_{2}\left(\alpha_{1}<\alpha_{2}\right)$ of the function $g_{x}(t)$. Obviously, $1<\alpha_{1}<e<\alpha_{2}$. Since $g_{x}(t)$ is increasing, $1<x<\alpha_{1}$, and we check by the math induction that $a_{1}(x)<a_{2}(x)<\cdots<a_{n}(x)<a_{1}$, so the limit of the sequence $\left\{a_{n}(x)\right\}$ exists and is just a fixed point $\alpha_{1}$.

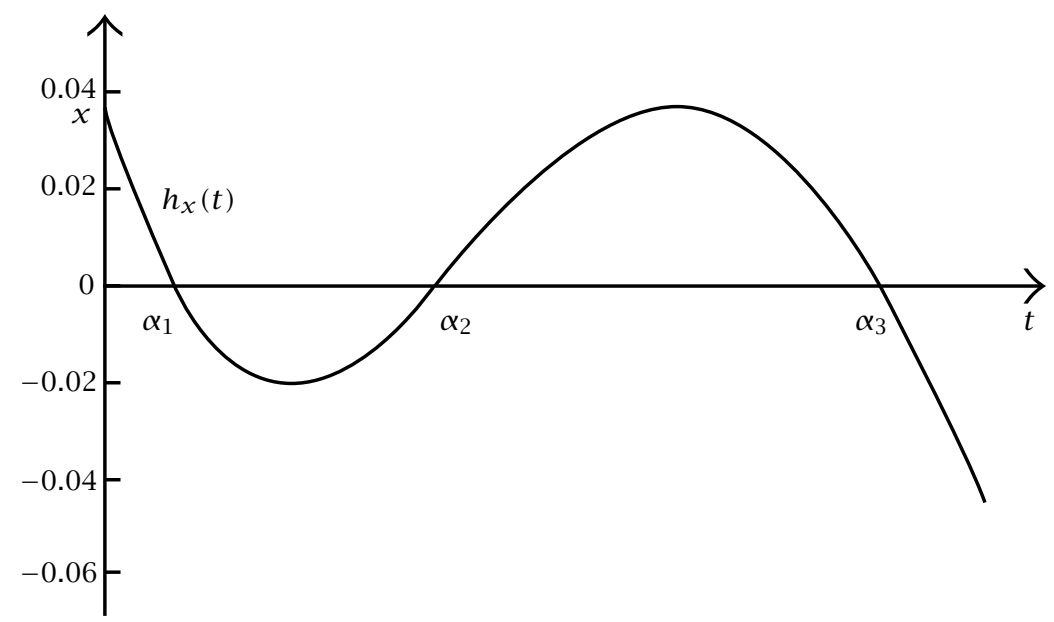

FIGURE 2.2. 


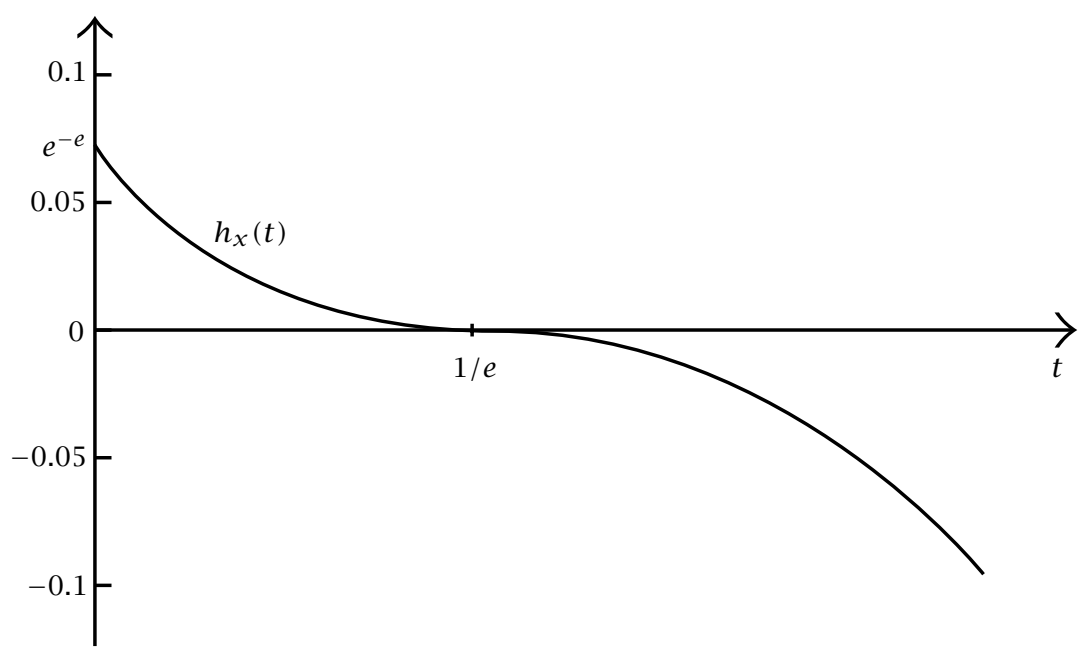

FIGURE 2.3.

CASE $5\left(x=e^{1 / e}\right)$. As before, $1<x<e$ and $\left\{a_{n}(x)\right\}$ is increasing and bounded by $e$, thus convergent to the only fixed point of $g_{e^{1 / e}}(t)$, namely $e$ itself.

CASE $6\left(x>e^{1 / e}\right)$. By Corollary 2.3, no fixed point of $g_{x}(t)$ exists, so the sequence $\left\{a_{n}(x)\right\}$ does not converge. Now, the proof is complete.

We note that in Case 4 of the above proof, $\alpha_{1}$ is an attracting fixed point, while $\alpha_{2}$ is repelling fixed point for $g_{x}(t)$. In the limiting Case 5 , the point $\alpha=e$ is left

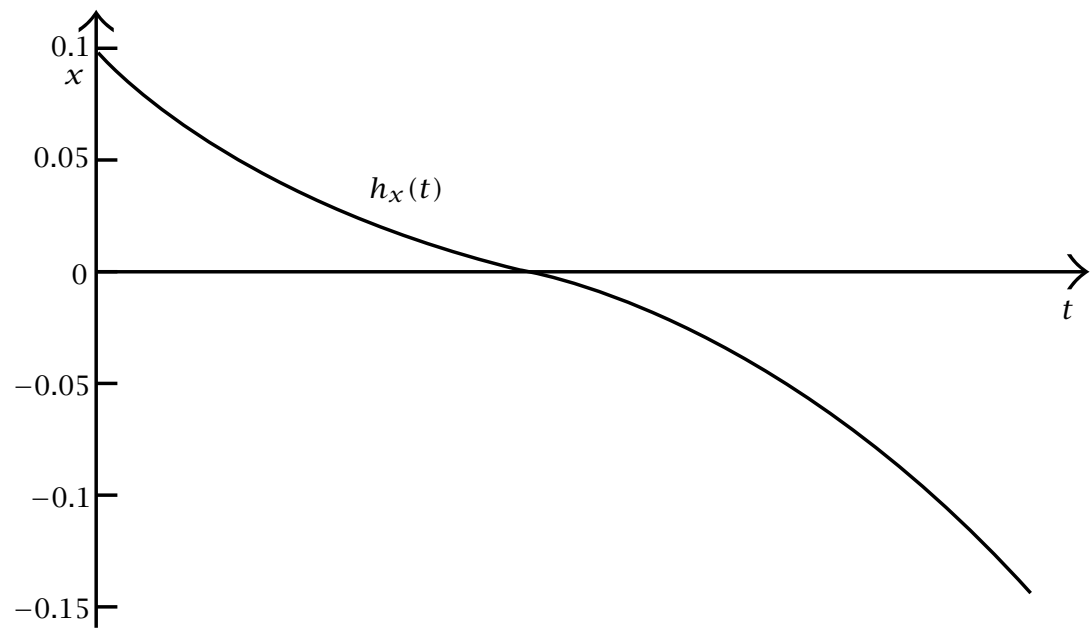

FIGURE 2.4. 
attracting and right repelling. Our Corollary 2.3 and Lemma 2.4 have some interesting relations to certain functional equations, one of them considered already by Euler (see Sierpiński [2]):

(a) $0<x<e^{-e}$. As we have already noticed, there exist exactly three zeros of the function $h_{x}(t)$, say, $\alpha_{1}<\alpha_{2}<\alpha_{3}$, so that $x^{x^{\alpha_{i}}}=\alpha_{i}$ for $i=1,2,3$, see (2.1); $\alpha_{1}$ and $\alpha_{3}$ are points of period 2 of the function $g_{x}(t)$, i.e., $x^{\alpha_{1}}=\alpha_{3}$ and $x^{\alpha_{3}}=\alpha_{1}$; $\left(\alpha_{2}\right.$ is just the fixed point of the function $g_{x}(t)$ ). Thus,

$$
\alpha_{1}^{\alpha_{1}}=\left(x^{\alpha_{3}}\right)^{\alpha_{1}}=\left(x^{\alpha_{1}}\right)^{\alpha_{3}}=\alpha_{3}^{\alpha_{3}} .
$$

In other words, $\alpha_{1}$ and $\alpha_{3}$ satisfy the functional equation $u^{u}=v^{v}$. For a detailed account on solving this equation we refer to Bomberger [1]. Observe that all $\alpha_{i}$ 's are in $(0,1)$.

(b) $1<x<e^{1 / e}$. As we noted after Lemma 2.2, there exist two distinct fixed points $\alpha_{1}$ and $\alpha_{2}$ of $g_{x}(t)$, and when $x \rightarrow 1^{+}, \alpha_{1} \rightarrow 1^{+}$, and $\alpha_{2} \rightarrow+\infty$. Since $x^{\alpha_{1}}=\alpha_{1}$ and $x^{\alpha_{2}}=\alpha_{2}$, we get

$$
\alpha_{1}^{\alpha_{2}}=\left(x^{\alpha_{1}}\right)^{\alpha_{2}}=\left(x^{\alpha_{2}}\right)^{\alpha_{1}}=\alpha_{2}^{\alpha_{1}},
$$

thus, $\alpha_{1}$ and $\alpha_{2}$ are related by the equation $u^{v}=v^{u}(u, v>0)$, called the Euler equation.

(c) $e^{-e} \leq x \leq 1$. Now, $\alpha_{1}$ and $\alpha_{3}$ considered in (a) collapse to the same point and both types of equations considered in (a) and (b) are automatically satisfied.

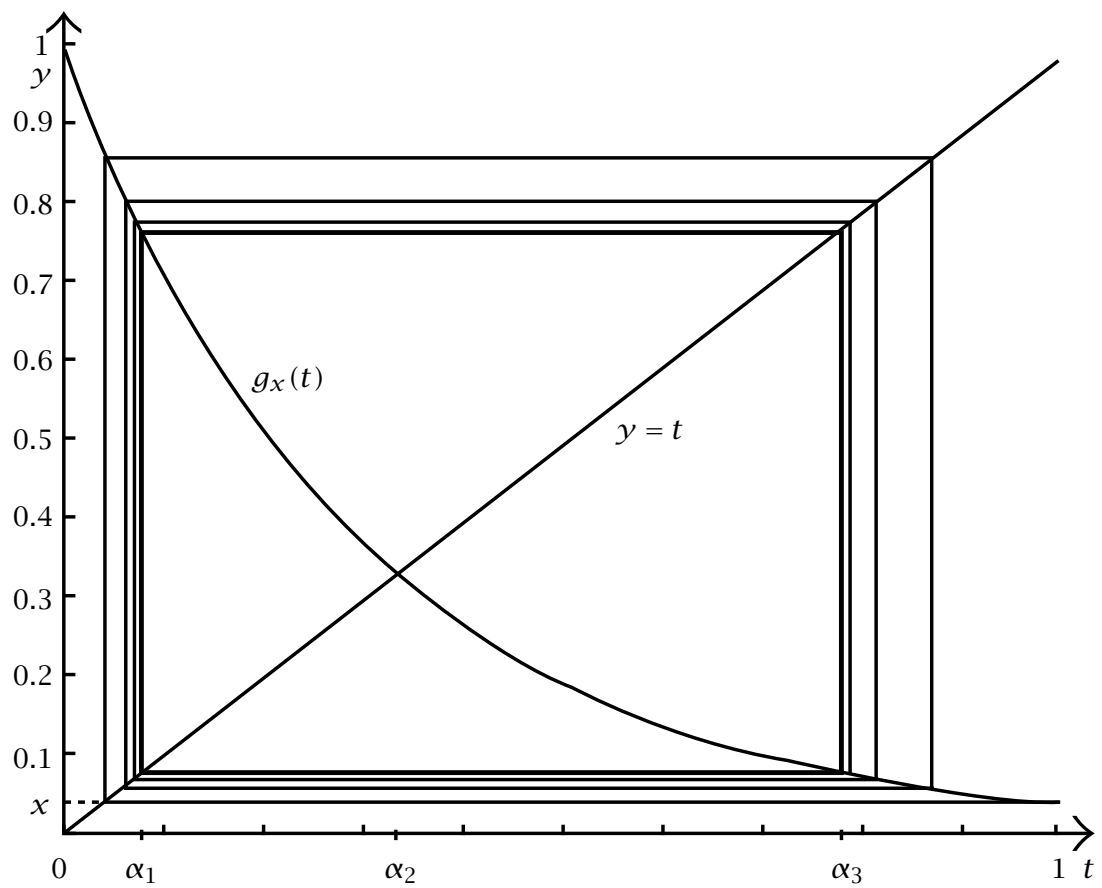

FIGURE 2.5 . 
For the sake of completeness of this exposition we describe the set of solutions of the Euler equation.

Proposition 2.5. The set of solutions of the Euler equation $u^{v}=v^{u}$, where $u \neq$ $v, u, v>0$ is given by a parametrized family $\left\{(u, v)=\left(\alpha^{1 /(\alpha-1)}, \alpha^{\alpha /(\alpha-1)}\right): \alpha>1\right\}$.

Proof. We note that if $0<u<v \leq 1$, then $u<u^{v}<u^{u}<v^{u}$, and a pair $(u, v)$ cannot be a solution. Similarly, when $0<u \leq 1<v$. Observe that $\ln (u) / u=\ln (v) / v$, and if this common value is less than $1 / e$, by Lemma 2.2 there exist exactly two solutions $u$ and $v$ of this equation and $1<u<e<v$. We postulate a solution in the form $u=t$ and $v=t^{\alpha}$, where $t>1$ and $\alpha>0$ are parameters. Thus,

$$
t^{t^{\alpha}}=\left(t^{\alpha}\right)^{t}=t^{\alpha t}
$$

so $t^{\alpha}=\alpha t$ and, in consequence, $t=\alpha^{1 /(\alpha-1)}$. Hence, $(u, v)=\left(\alpha^{1 /(\alpha-1)}, \alpha^{\alpha /(\alpha-1)}\right)$. For any $1 \neq \alpha>0, \alpha^{1 /(\alpha-1)}>1$, so in order to keep $u<v, \alpha$ must be greater than 1 . We check that the pair $(u, v)$ actually solves the equation:

$$
u^{v}=\left(\alpha^{1 /(\alpha-1)}\right)^{\alpha^{\alpha /(\alpha-1)}}=\alpha^{1 /(\alpha-1) \alpha^{\alpha /(\alpha-1)}}=\alpha^{1 /(\alpha-1) \alpha^{1+1 /(\alpha-1)}}=\left(\alpha^{\alpha /(\alpha-1)}\right)^{\alpha^{1 /(\alpha-1)}}=v^{u} .
$$

Now, we show that this parametrized family exhausts all possible solutions of the Euler equation such that $u<v$. In fact, if $\alpha \rightarrow 1^{+}, \alpha^{1 /(\alpha-1)} \rightarrow e$, and $\alpha^{\alpha /(\alpha-1)} \rightarrow e$; if $\alpha \rightarrow$ $+\infty, \alpha^{1 /(\alpha-1)} \rightarrow 1$, and $\alpha^{\alpha /(\alpha-1)} \rightarrow+\infty$. The result now follows from the intermediate value theorem.

ACKNOWLEDgements. We are very grateful to Dr. Denny Gulick from the University of Maryland, College Park and an anonymous referee whose suggestions and comments were invaluable for improving the presentation of this paper. This research was supported by the Fall 1997 MIE Faculty Grant.

\section{REFERENCES}

[1] J. D. Bomberger, On the solutions of $a^{a}=b^{b}$, Pi Mu Epsilon J. 9 (1993), no. 9, 571-572. Zbl 875.26013.

[2] W. Sierpiński, Elementary Theory of Numbers, 2nd ed., Elsevier Science Publ. Co., New York, 1973.

Roman SZnajder: Department of Mathematics, Bowie State University, Bowie, MaryLAND 20715, USA

E-mail address: rsznajde@bowi estate.edu

Kanchan Basnyat: Department of Computer SCience, Bowie State UniVersity, Bowie, MARYLAND 20715, USA

E-mail address: kbasnyat@cs . bowi estate.edu 


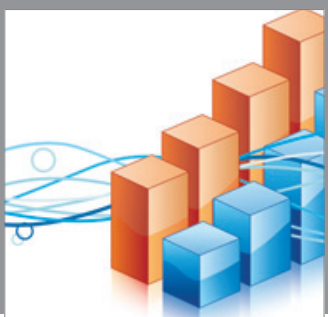

Advances in

Operations Research

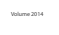

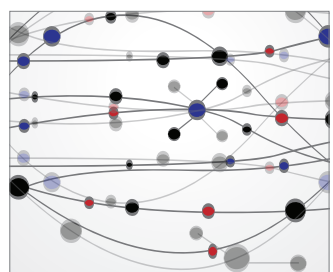

\section{The Scientific} World Journal
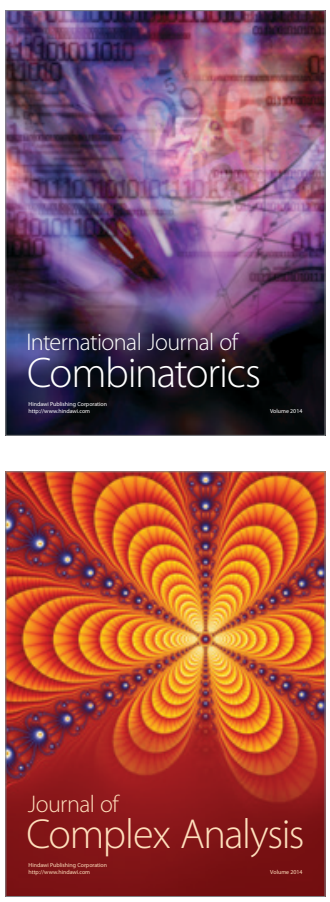

International Journal of

Mathematics and

Mathematical

Sciences
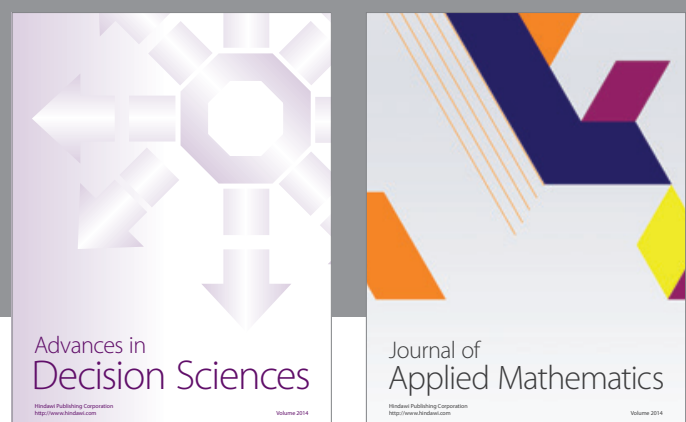

Journal of

Applied Mathematics
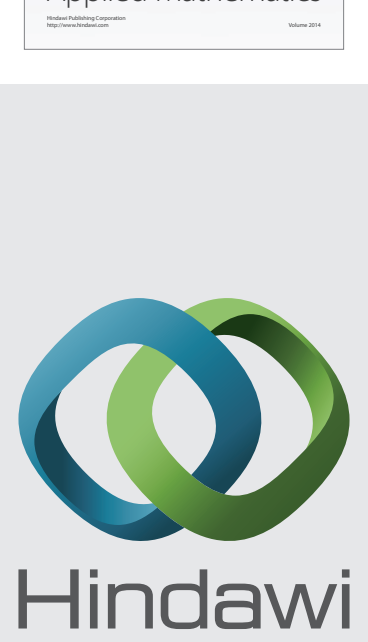

Submit your manuscripts at http://www.hindawi.com
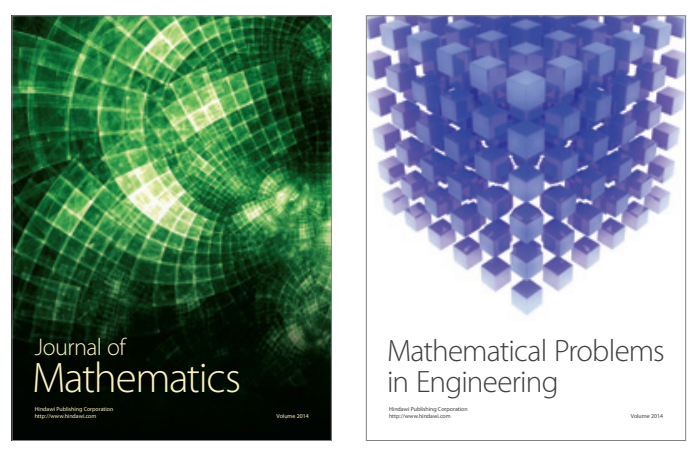

Mathematical Problems in Engineering
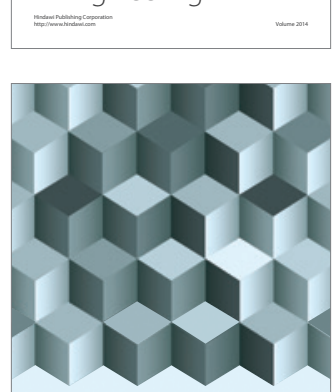

Journal of

Function Spaces
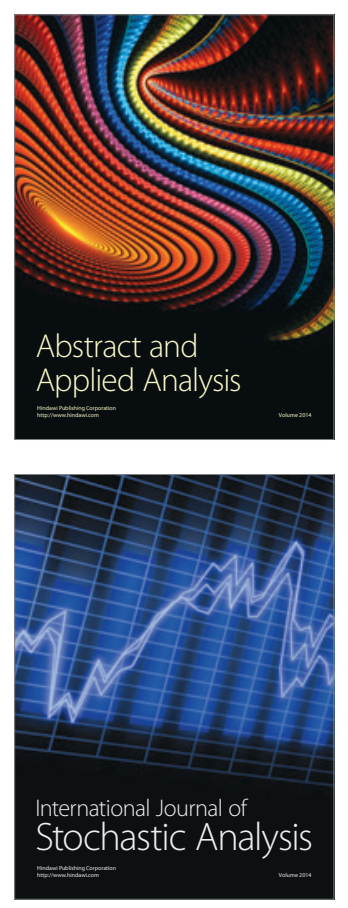

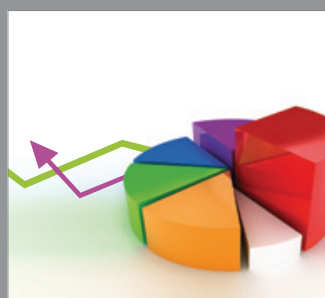

ournal of

Probability and Statistics

Promensencen
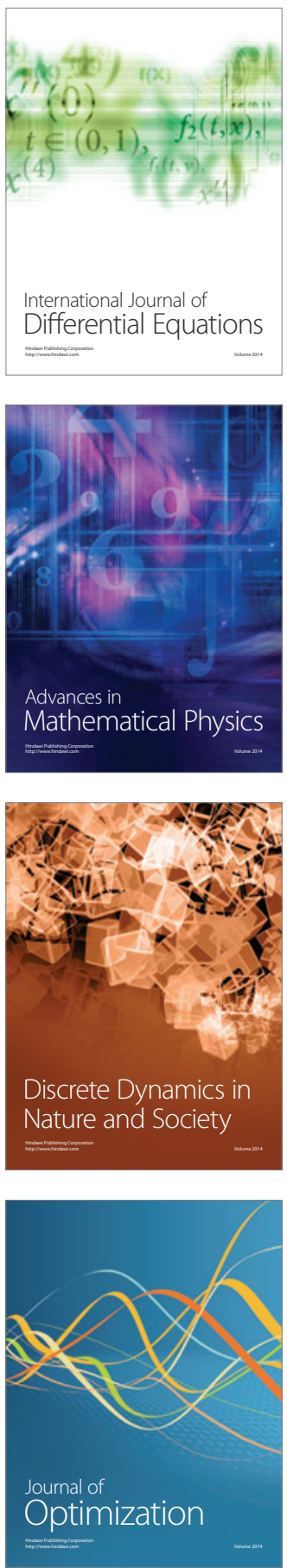